\title{
Utility of Next Generation Sequencing in Genetic Testing and Counseling of Disorders Involving the Musculoskeletal System-Trends Observed From a Single Genetic Unit
}

\section{Gayatri R lyer}

Kamineni Academy of Medical Sciences and Research Centre

Roshan Kumar

Kamineni Academy of Medical Sciences and Research Centre

\section{Subhadra Poornima}

Kamineni Academy of Medical Sciences and Research Centre Aruna Priya

Kamineni Academy of Medical Sciences and Research Centre

Keerthi Juturu

Kamineni Academy of Medical Sciences and Research Centre

\section{Lekhanga Bhatnagar}

Kamineni Academy of Medical Sciences and Research Centre

\section{Srinka Arora}

Kamineni Academy of Medical Sciences and Research Centre

\section{Vaishnavi Suresh}

Kamineni Academy of Medical Sciences and Research Centre

\section{Prashanth Utage}

Kamineni Academy of Medical Sciences and Research Centre

\section{Sarah Bailur}

Kamineni Academy of Medical Sciences and Research Centre

\section{Akhilesh N Pujar}

LVPEI: LV Prasad Eye Institute

Qurratulain Hasan ( $\nabla$ qhasan2000@yahoo.com )

Kamineni Academy of Medical Sciences and Research Centre

\section{Research article}

Keywords: musculoskeletal disorders, skeletal dysplasia, next generation sequencing, genetic counseling, molecular diagnosis 
Posted Date: April 27th, 2021

DOl: https://doi.org/10.21203/rs.3.rs-431437/v1

License: (c) (i) This work is licensed under a Creative Commons Attribution 4.0 International License. Read Full License

Version of Record: A version of this preprint was published at Journal of Orthopaedic Surgery and Research on February 5th, 2022. See the published version at https://doi.org/10.1186/s13018-02202969-x. 


\section{Abstract}

Background: Disorders involving the musculoskeletal system are often identified with short stature and a range of orthopedic problems. The clinical and genetic heterogeneity of these diseases, with several characteristic overlaps, makes specific diagnosis difficult for clinicians using conventional tests, hence molecular testing becomes essential for appropriate diagnosis and management.

Methods: Next Generation Sequencing (NGS) based testing has become common in current day clinical practice. To do genetic counseling in cases with disorders in musculoskeletal system, classify and refer for appropriate genetic testing and to assess utility of NGS based testing for molecular diagnosis. Detailed pre and posttest genetic counseling, NGS based clinical exome testing, genotype-phenotype correlation, and segregation analysis.

Results: This manuscript reports a molecular spectrum of variants in 34 orthopedic cases referred to a single genetic unit attached to a tertiary care hospital. The diagnostic yield of NGS based tests coupled with genetic counseling and segregation analysis was $79 \%$ which included 7 novel variants. Cases which benefited or will get a benefit in the near future were 53\% (i.e. 18/34 cases), as eight underwent prenatal diagnosis, since they were either in their early gestation or had planned a pregnancy subsequent to molecular testing, while ten cases were premaritally/prenatally counseled for the families to take informed decisions as they were in the reproductive age.

Conclusions: The report highlights the importance of NGS based tests even in a low resource setting as it helps patients, families and health care providers in reducing the economic, social and emotional burden of these disorders.

\section{What's Known?}

Next generation sequencing is a tool which has come into mainstream clinical practice in developed nations. Genetic counseling helps clinicians, patients and families understand the ailments and manage it appropriately

\section{What's new?}

The article demonstrates utility of next generation sequencing in Indian population and illustrates the importance in using the test for optimal benefits in clinical management, surveillance, prenatal diagnosis and eventually bringing down morbidity and mortality of musculoskeletal disorders. Genetic counseling and follow up testing increased diagnostic yield to $79 \%$, the highest in musculoskeletal disorders reported earlier. The manuscript also reports 7 novel variants associated with these disorders

\section{Introduction}


Disorders involving the musculoskeletal system (DMS) are generalized abnormalities of the muscle and skeletal system often marked by short stature and a spectrum of mobility and orthopedic problems ${ }^{1}$. There are more than 450 disorders that involve the DMS and can be clustered into 40 different groups defined by clinical, molecular, biochemical, and/or radiographic criteria. It is estimated that $5 \%$ of cases with congenital birth defects belong to the category of skeletal dysplasia ${ }^{2}$ and some of them can be diagnosed prenatally by ultrasound 1,3 . The clinical and genetic heterogeneity of these diseases, with several characteristic overlaps, makes specific diagnosis difficult for Pediatricians, Orthopaedicians and Radiologists using conventional modalities, hence molecular diagnostics becomes essential.

DMS predominantly affects the muscle, cartilage or skeletal system and are associated with several genetic syndromes. Patients with these disorders in India report either to pediatric, neurology or orthopedic departments with skeletal abnormalities, altered gait, short stature or joint pain. Disability support in low resource countries including India is abysmally low ${ }^{4}$ and families with an affected child face several problems in day-to-day life, if more than one family member is affected then their social, financial, and emotional troubles are aggravated ${ }^{5}$. Identifying the type of genetic inheritance followed by appropriate genetic counselling helps families understand the problem and take informed decisions. Based on pedigree analysis type of inheritance can be deduced in some cases like autosomal dominant $(A D)$, autosomal recessive $(A R)$, or $X$-linked dominant $(X D)$ and recessive forms $(X R)$ depending on the location of the associated genes ${ }^{6}$. Confirmation for this again comes after molecular diagnosis.

Since many of the DMS have overlapping features, with heterogeneous symptoms it is clinically difficult to make an accurate diagnosis. In a developing country like India, about $80 \%$ of the population has to cater to their own medical needs for consultation, diagnosis as well as treatment. A delayed diagnosis would put an additional burden on increased consultations, cross consultations and repeated diagnostic testing. Even with an absolute clinical diagnosis, reproductive decisions cannot be made, thus NGS based testing though relatively expensive than other biochemical or radiological test, is a one-time investment in facilitating streamlined syndrome follow up and reproductive decision making. In the present study we evaluated the role of NGS in diagnosing patients with musculoskeletal abnormalities referred to the Genetics unit of a tertiary care hospital in South India with comprehensive pre and posttest genetic counseling to help patients and their families in taking informed healthcare and reproductive decisions.

\section{Methods}

During the period January 2015 to August 2019, 70 cases with DMS were referred to the department of Genetics and Molecular Medicine, Kamineni Hospitals, Hyderabad for genetic testing and counseling. Clinical, social, and family history of all the cases was recorded in a well-designed proforma by a genetic counselor after a face-to-face interview with patients or parents/guardians of the affected individuals. Of the total cases, 36 were evaluated with diagnostic modalities like karyotyping, multiple ligation probe amplification assays and microarray, especially those cases with a clinical diagnosis of neuromuscular disorders like Duchenne muscular dystrophy and spinal muscular atrophy. The remaining 34 cases were 
deemed appropriate based on clinical features and family history for carrying out clinical exome with NGS. These included two products of conception (POC), which was abortuse material, a case of neonatal death and three couples who were analyzed for carrier status in view of bad obstetric history with radiological evidence of skeletal deformities with or without other congenital anomalies in the fetus. Informed consent was taken from patients/parents/guardians prior to obtaining $2 \mathrm{ml}$ of peripheral blood in EDTA or POC tissue as per the Institutional Ethics Committee of Kamineni Hospitals (Registration\#ECR/ 58/Inst/AP/2013) guidelines. This study was carried out in accordance with the recommendations of International Council of Harmonization and Good Clinical Practice. All subjects/families gave written informed consent in accordance with the Declaration of Helsinki

The samples were outsourced to different commercial laboratories at case per case basis depending upon the cost and turn-around time. Major criteria of choosing the testing lab was that they analyzed 300 genes currently recommended for DMS and followed standard protocol ${ }^{7}$. Reporting was done in accordance with the American College of Medical Genetics and Genomics and the Association for Molecular Pathology guidelines ${ }^{8}$

\section{Results}

The age range of cases was 2 days to 56 years and age of onset of symptoms was from in-utero to 15 years. Affected individuals were 21 males and 10 females making the male to female ratio $2: 1$ (65.51\% males and $34.48 \%$ females). Three couples assessed were not included in the ratio calculation.

Pedigree analysis indicated that of the 34 cases, 15 (44.11\%) had positive family history with a first/second degree relative who had same or similar disorder, while 19 cases (55.88\%) were sporadic. From the pedigree, we estimated five cases to be AD, of which four were confirmed, while the fifth one was an XD disorder confirmed after NGS testing. Fifteen cases were predicted to have AR inheritance, of which 14 were confirmed by the molecular report, whereas the last case was identified to have an XR condition in a child. Only one pedigree showed an XR which was confirmed after molecular testing. Consanguinity was documented in $15(44.11 \%)$ families suggesting AR inheritance, however, only 12 out of 15 consanguineous cases had a confirmed AR disorder post molecular diagnosis (Tables 1 and 2). AD cases identified were 15, XR were 3 and one family was diagnosed to have XD disorder.

\section{Clinical spectrum of the cases}

The cases which were evaluated by NGS were broadly classified into disorders involving musculoskeletal system and storage disorders depending on whether or not they showed systemic involvement.

Disorders involving Musculoskeletal system - $76 \%$ (26 of the 34 ) cases were identified as . musculoskeletal disorders with genetic basis after radiological and clinical criteria and were categorized as:

\section{a. Osteogenesis Imperfect (OI)}


Osteogenesis imperfecta $(\mathrm{OI})$ is a genetic disorder affecting the connective tissue and is mainly characterized by extremely fragile/brittle bones that fracture with trivial traumas ${ }^{9}$. Ol is of more than ten types based on clinical features that often overlap. Severity varies with type I being the mildest form and type II being the severest form. COL1A1 and COL1A2 gene mutations are responsible for more than $90 \%$ of all cases of OI, however, other genes have also been associated ${ }^{10}$.

The eight cases (1.1.1-1.1.8 cases) clinically diagnosed as Ol had common features such as recurrent fractures and blue sclera. Their age of disease onset was from in-utero to 15 years. Seven of the patients were born of non-consanguineous marriage and four of these had another family member affected (Table 1.1). Four out of eight cases were identified (50\% diagnostic yield) with pathogenic heterozygous sequence variant in COL1A1 or COL1A2 genes indicating AD inheritance. A homozygous intronic variant in COL1A2 gene which is known to be polymorphic and may be associated with phenotype was found in a 5-year-old, with childhood onset recurrent fractures, blue sclera, and a positive family history. Two cases were identified with homozygous Variant of uncertain significance (VUS) in COL1A1 gene and SERPINF1 gene, it is interesting to note that the case 1.1.5 with COL1A1 who had homozygous VUS also exhibited additional clinical features like dentogenesis imperfecta, slurred speech, dysmorphism and mild scoliosis indicating the severity likely caused due to homozygosity. Segregation analysis may be useful to understand the significance of these variants. However, no significant variant correlating with phenotype was identified in case 1.1.8.

\section{b. Osteopetrosis}

Osteopetrosis is a rare genetic disorder characterized by an increase of bone mass due to defective osteoclast function. Patients typically display spontaneous fractures, anemia, and in the most severe forms hepatosplenomegaly and compression of cranial facial nerves leading to deafness and blindness

11. We confirmed diagnosis of two cases of osteopetrosis (1.2.1-1.2.2). A heterozygous pathogenic variant in CLCN7 gene indicating Osteopetrosis type 2 (1.2.1) and the other, a VUS in LRP gene known to cause Osteopetrosis type 1 (case 1.2.2)

\section{c. Split-hand/foot malformation-6}

Split Hand/Split Foot Malformation or Ectrodactyly is a genetic disorder characterized by the complete or partial absence of some fingers or toes, often combined with clefts in the hands or feet ${ }^{12}$. Two sisters (1.3 case) with ectrodactyly born to consanguineous parents were identified with homozygous pathogenic variant in WNT10B gene confirming the diagnosis of split hand/foot malformation 6.

\section{d. Skeletal dysplasia with in-utero presentation}

It is well established that DMS can be identified in the prenatal period. Two POC, one perinatal death and three couple samples were assessed in this category. Couple samples were analysed because of $\mathrm{BOH}$ with skeletal involvement and unavailability of samples from the affected fetus. Analysis of the male fetal sample (case 1.4.1) revealed hemizygous VUS in MTM1 gene diagnostic of XR myotubular 
myopathy, which is associated with skeletal dysplasia and polyhydramnios in utero, both characteristics, were reported in the ultrasound report available ${ }^{13}$. Since the couple was planning their next child, the mother was evaluated and was identified to have the same variant in heterozygous state confirming carrier status. This also explained the childhood death of two maternal uncles which was documented in the pedigree. Hence this MTM1c.413C>T p.Thr138Met variant can be considered as a novel pathogenic variant after confirmation with functional analysis.

The second POC (case 1.4.2) revealed a novel pathogenic homozygous variant c.867_877del causing protein truncation p.GIn289HisfsTer2 in CUL7 gene causative of 3M syndrome, which has a phenotype of severe prenatal and postnatal growth retardation, long, slender tubular bones, reduced antero posterior diameter of the vertebral bodies and delayed bone age ${ }^{14}$. While the ample from a neonate who passed away (case 1.4.3) with a clinical diagnosis of Dandy walker with radiological evidence of malformation and oligohydramnios exhibited two compound heterozygous VUS (which included novel variant c.280+7_280+9AG) in POMT1 gene causative of Walker-Wurburg syndrome ${ }^{15}$.Segregation analysis was recommended in both cases

Three couples were screened by NGS in view of consanguinity and BOH with DMS. Two couples were identified to be carriers for AR disorders with single heterozygous causative variant whereas one couple had different causative variants in same gene contributing to compound heterozygosity in offspring. Case 1.4.4 was identified with compound heterozygosity comprising of a novel pathogenic (c.6134C>T, p.Ser2045Leu) variant and VUS in CEP290 associated with Joubert syndrome 5 (OMIM \# 610 188). Case 1.4.5 revealed heterozygosity in both partners for likely pathogenic variant in RBBP8 causative of Seckel Syndrome 2 (OMIM \# 606744) whereas case 1.4.6 revealed both parents to be heterozygous carriers for a VUS in COL11A2 associated with Fibrochondrogenesis 2 (OMIM \# \# 614524).

\section{e. Short stature}

Although short stature is observed in most skeletal dysplasia cases, four of our syndromic cases were found to have short stature in addition to other features. A female member of a family where multiple members had renal rickets was tested and identified to have a pathogenic variant in PHEX gene confirming the diagnosis of $\mathrm{X}$ linked dominant Hypophosphatemic rickets ${ }^{16}$.

Other three pediatric cases had short stature as a part of a developmental disorders, a homozygous pathogenic variation in ERC66 gene associated with Cockayne syndrome $\mathrm{B}^{17}$, cerebro oculofacio skeletal syndrome 1 was identified in case 1.5.2, while the third case exhibited VUS in CUL4B gene causative of Cabezas type of X-linked syndromic mental retardation ${ }^{18}$ and the fourth case was identified to have heterozygous VUS in AHDC1 associated with Xia Gibbs Syndrome ${ }^{19}$.

\section{f. Joint stiffness and pain}

Two cases of arthritis as a part of systemic disorders were evaluated in this study. Case 1.6.1 of 21 year old male with clubbing of digits, Erlenmeyer flask deformity of distal tibia and fibula, thrombocytopenia 
and bilateral polycystic kidneys was identified to have a novel homozygous pathogenic variant c.325delG causing protein truncation p.Ala109LeufsTer64 in SLCO2A1 gene diagnosing it as primary hypertrophic osteoarthropathy, autosomal recessive 2 (PHOAR2) ${ }^{20}$.It is interesting to note that the same individual was also identified with a heterozygous pathogenic variant in PKD2 gene c.637C>T p.Arg213Ter associated with $A D$ polycystic kidney disease explaining the renal phenotype. Case 1.6.2 was identified with a pathogenic homozygous variant in CCN6 gene causative of autosomal recessive pseudorheumatoid dysplasia manifesting as joint stiffness since the young age of 16 years ${ }^{21}$.

A female who had joint restrictions and genu valgum and avascular necrosis was identified to have a pathogenic variant in COL9A1 gene associated with multiple epiphyseal dysplasia ${ }^{22}$. A 8 months old male born to non-consanguineous parents presented with hyperpigmentation and joint swellings and was reported to have novel likely pathogenic duplication c.54_60dupGCTGTGG causing premature truncation (p.Leu21Alafs ${ }^{\star 26}$ ) in ANTXR2 genecausative of hyaline fibromatosis, a condition where there is deposition of amorphous hyaline material in skin and visceral organs ${ }^{23}$. A 30 year old female with family history of cancer who has café au lait spots was assessed and found to have a variant in NF1 gene associated with neurofibromatosis ${ }^{24}$.

\section{Storage disorders}

Lysosomal storage disorders (LSDs) are a large group of more than 50 different inherited metabolic diseases which, in the great majority of cases, result from the defective function of specific lysosomal enzymes and non-enzymatic lysosomal proteins or non-lysosomal proteins involved in lysosomal biogenesis 25 .

\section{a. Mucopolysaccharidoses (MPS)}

MPS is characterized to seven subtypes and are caused by deficiencies in the lysosomal enzymes necessary for the degradation of glycosaminoglycans (GAGs). Storage of GAGs affects the skeletal tissue, cartilage and connective tissues, as well as the peripheral and central nervous system ${ }^{26}$.

Of the five cases clinically suspected with MPS, three were consanguineous. Pathogenic variations in IDUA were identified in two cases diagnosing MPS I. A novel hemizygous likely pathogenic missense variation c.1493 $\mathrm{G}>\mathrm{C}$ causing protein change p.Arg498Thr was identified in IDS gene in a $2 \mathrm{yr} / \mathrm{male}$ patient confirming MPS II. Two brothers with knock knees and h epatosplenomegaly had a likely pathogenic homozygous variant in GALNS gene which is associated with MPS IVA. Another 7-year-old male with intellectual disability, corneal clouding was identified to have a homozygous VUS in GUSB gene causative of MPS VII. The younger brother of the proband was identified with the same variant in GUSB gene followed by enzyme analysis showing deficient $\beta$-Glucoronidase activity thereby confirming the pathogenicity of the VUS identified.

\section{b.Mucolipidosis}


Mucolipidosis has the clinical and biochemical features of both MPSs and sphingolipidoses, being characterized by the accumulation of glycoproteins and glycolipids ${ }^{26}$. In our cohort, there were two clinically cases suspected of mucolipidosis with consanguinity in one case. Pathogenic GNPTG homozygous variant was identified in a 7yr/female. Two VUSs in GNPTAB with compound heterozygosity were identified in another female child of 4 years. A 9-year-old female (2.2.3) born to nonconsanguineous couple with storage disorder like symptoms and elevated urine GAGs was suspected to have mucolipidosis in view of no likely causative variants in MPS genes, however, no diagnostic variant was identified to confirm mucolipidosis in this patient.

\section{Discussion}

A total of $94 \%(32 / 34)$ cases were identified with a variation in genes likely causative of the disorder, thereby establishing genotype - phenotype correlation. Of the sequence variants identified, 15 were pathogenic, 6 were likely pathogenic and 13 were variants of uncertain significance according to ACMG guidelines ${ }^{27}$.Seven novel variants associated with the conditions were identified. Twenty-one pathogenic and likely pathogenic variants were identified and segregation/carrier analysis of 6 out of the 13 VUS were confirmed as pathogenic (cases 1.1.7, 1.4.1, 1.4.3, 1.4.4, 1.5.3, 2.1.5) thus giving a total yield of $79 \%$ $(27 / 34)$ where the patient had definitive molecular diagnosis. Cases 1.1.8 and 2.2.3 were not identified with any clinically significant variants in the gene panel used. Hence, whole exome sequencing maybe required screening a larger panel of genes or identifying novel genes associated with the DMS.

Traditionally, healthcare services in India revolve around the specialist clinicians and patients have direct access to Pediatricians or Orthopedician without going through a primary physician. They are responsible for recommending health care interventions and for referring patients to the enetic units or ordering tests. Until recently, DMSs were diagnosed solely on biochemical and radiological findings and were rarely confirmed by specific gene mutation analysis ${ }^{25}$. A paper by Uttarilli et al (2019) described the shift from single gene testing to panel testing over a decade from multiple centers in India since in most cases large single gene Sanger testing is more expensive than NGS based panel testing with a better diagnostic yield 26. As compared to the reports from Manipal ${ }^{28}$ and Kerala ${ }^{29}$, we observed $44 \%$ consanguinity in our cases, against their $37 \%$ and $11.6 \%$, respectively. When considering only AR disorders, the rate of consanguinity was seen to be $80 \%$ which is significantly higher compared to Manipal data of $43 \%$ and $31 \%$ from the Kerala publication.

There is a requirement to have a definitive diagnosis for patients and families with genetic diseases to understand the course of the disease and available treatment/ management options to take informed healthcare decisions especially for MPS. Over the past few decades, remarkable advances in molecular diagnosis with the advent of affordable NGS tests make it possible to analyse several genes in a single disease-targeted panel test ${ }^{30}$. A boom in bioinformatics has further helped in interpreting NGS data efficiently especially in countries like India, where databases for variants are not available for their specific population ${ }^{31}$. The data generated will help us develop a disease specific gene panel unique for 
our population if all companies donate their raw data files into a common database. This will also help in focusing our research on studying the functional significance of reported VUS showing phenotype correlation and confirming if they are novel pathogenic variants for our population.

The NGS test was helpful in counselling ten premarital/pre-conceptional cases and eight families benefited as they could undergo prenatal diagnosis. Thus, with the current diagnostic yield, $53 \%$ of the families could take informed reproductive decisions based on NGS testing who are currently in their reproductive age, the other $26 \%$ are equipped with molecular variant information that will facilitate premarital and prenatal counseling and testing in the near future.

\section{Conclusion}

This study shows that NGS is an important tool for effective diagnosis of DMS even in a low resource country like India as its diagnostic yield is $79 \%$ after a proper pre-test and post-test counseling. The patients/families in our country usually consult different hospitals/clinicians in the hope of diagnosis/treatment, undergo repeated tests which disappoint them, many of them in the absence of diagnosis have another child with the same disease, which is devastating. Other members of their family may get affected as no preventative carrier testing was advised or they were not informed about prenatal testing. Also, syndrome specific management in MPS and similar cases could have been planned in affected individuals. All this can only be facilitated by a pre and posttest genetic counseling to educate families adequately after a molecular test. This exercise would be instrumental in taking informed reproductive decisions significantly reducing the morbidity and mortality burden on families and society.

\section{List Of Abbreviations}

NGS- Next Generation Sequencing

DMS-Diseases associated with musculoskeletal systems

AR: Autosomal Recessive

AD: Autosomal Dominant

XD: X-Linked Dominant

XR: X-Linked Recessive

OI: Osteogenesis Imperfecta

LSD: Lysosomal storage disorders

POC: Products of Conception

MPS: Mucopolysaccharidoses 
VUS: Variant of Uncertain Significance

\section{Declarations}

\section{Ethics approval and consent to participate:}

The study received approval from Institutional Ethics Committee of Kamineni Hospitals

(Registration\#ECR/ 58/Inst/AP/2013). Study was carried out in accordance with the recommendations of International Council of Harmonization and Good Clinical Practice. All subjects/families gave written informed consent in accordance with the Declaration of Helsinki

Consent for publication: Not Applicable

Availability of data and materials: Any additional information can be shared upon request

Competing interests: The authors declare there are no competing interests.

Funding: This research did not receive any specific grant from funding agencies in the public, commercial, or not-for-profit sectors

Authors' contributions: GRI wrote the first draft of the manuscript, RK, PRU and SB reviewed and curated the clinical information, APK, SP, KKJ,LB, SA and ANP compiled patient information and prepared the tables, QH supervised the project and did scientific editing of the manuscript. All authors reviewed the manuscript.

Acknowledgement: Patients and families. Staff and students of Department of Genetics \& Molecular Medicine and Orthopedics, Kamineni Hospitals, Hyderabad.

\section{References}

1. Alanay, Y. and Lachman, R.S., (2011). A review of the principles of radiological assessment of skeletal dysplasias. Journal of clinical research in pediatric endocrinology, 3(4), p.163.

2. Gnoli, M., Pedrini, E., Mordenti, M., Tremosini, M. and Sangiorgi, L., (2014).. Skeletal dysplasias: approach to the clinical diagnosis and implication of appropriate diagnosis for knowledge and research studies in these rare diseases. Hereditary multiple Osteochondromas as example/paradigm. In Italian journal of pediatrics40(1), pp. 1-2. BioMed Central.

3. Parilla BV, Leeth EA, Kambich MP, Chilis P, MacGregor SN. (2003) Antenatal detection of skeletal dysplasias. Journal of ultrasound in medicine. 22(3):255-8.

4. Ministry of Statistics and Programme Implementation, Government of India Report on Persons with Disabilities in India. (2018), NSS Report no. 583 (76/26/1)

5. Golics, C.J., Basra, M.K.A., Finlay, A.Y. and Salek, S., (2013). The impact of disease on family members: a critical aspect of medical care. Journal of the Royal Society of Medicine, 106(10),399- 
407.

6. Krakow D. Skeletal dysplasias. Clinics in perinatology. (2015) ;42(2):301-19.

7. De Groot LJ, Chrousos G, Dungan K, Feingold KR, Grossman A, Hershman JM, Koch C, Korbonits M, McLachlan R, New M, Purnell J. Endotext [Internet].

8. Zhytnik, L., Maasalu, K., Reimann, E., Prans, E., Kõks, S. and Märtson, A., (2017). Mutational analysis of COL1A1 and COL1A2 genes among Estonian osteogenesis imperfecta patients. Human genomics, 11(1),

9. Coudert, A.E., de Vernejoul, M.C., Muraca, M. and Del Fattore, A., (2015). Osteopetrosis and its relevance for the discovery of new functions associated with the skeleton. International journal of endocrinology.

10. Van Buggenhout, G. and Fryns, J.P., (2003). The NORD Guide to Rare Disorders.

11. Amburgey, K., Tsuchiya, E., de Chastonay, S., Glueck, M., Alverez, R., Nguyen, C.T., Rutkowski, A., Hornyak, J., Beggs, A.H. and Dowling, J.J., 2017. A natural history study of X-linked myotubular myopathy. Neurology, 89(13), 1355-1364.

12. Huber, C., Delezoide, A.L., Guimiot, F., Baumann, C., Malan, V., Le Merrer, M., Da Silva, D.B., Bonneau, D., Chatelain, P., Chu, C. and Clark, R., (2009). A large-scale mutation search reveals genetic heterogeneity in 3M syndrome. European journal of human genetics, 17(3).395-400.

13. Huber C, Delezoide AL, Guimiot F, Baumann C, Malan V, Le Merrer M, Da Silva DB, Bonneau D, Chatelain P, Chu C, Clark R(2009). A large-scale mutation search reveals genetic heterogeneity in $3 \mathrm{M}$ syndrome. European journal of human genetics:395-400.

14. Ruppe MD. X-linked hypophosphatemia. InGeneReviews®[Internet] (2017). University of Washington, Seattle.

15. Wang, X., Huang, Y., Yan, M., Li, J., Ding, C., Jin, H., Fang, F., Yang, Y., Wu, B. and Chen, D., (2017). Molecular spectrum of excision repair cross-complementation group 8 gene defects in Chinese patients with Cockayne syndrome type A. Scientific reports, 7(1), 1-9.

16. Okamoto, N., Watanabe, M., Naruto, T., Matsuda, K., Kohmoto, T., Saito, M., Masuda, K. and Imoto, I., (2017). Genome-first approach diagnosed Cabezas syndrome via novel CUL4B mutation detection. Human genome variation, 4(1), 1-3.

17. Murdock, D.R., Jiang, Y., Wangler, M., Khayat, M.M., Sabo, A., Juusola, J., McWalter, K., Schatz, K.S., Gunay-Aygun, M. and Gibbs, R.A., (2019). Xia-Gibbs syndrome in adulthood: a case report with insight into the natural history of the condition. Molecular Case Studies, 5(3), .a003608.

18. Zhang, Z., He, J.W., Fu, W.Z., Zhang, C.Q. and Zhang, Z.L., (2013). Mutations in the SLCO2A1 gene and primary hypertrophic osteoarthropathy: a clinical and biochemical characterization. The Journal of Clinical Endocrinology \& Metabolism, 98(5).E923-E933.

19. Bhavani, G.S., Shah, H., Shukla, A., Dalal, A. and Girisha, K.M., (2015). Progressive pseudorheumatoid dysplasia. In GeneReviews $₫$ [Internet]. University of Washington, Seattle. 
20. Jackson, G.C., Mittaz-Crettol, L., Taylor, J.A., Mortier, G.R., Spranger, J., Zabel, B., Le Merrer, M., Cormier-Daire, V., Hall, C.M., Offiah, A. and Wright, M.J., (2012). Pseudoachondroplasia and multiple epiphyseal dysplasia: a 7-year comprehensive analysis of the known disease genes identify novel and recurrent mutations and provides an accurate assessment of their relative contribution. Human mutation, 33(1),144-157.

21. Mantri, M.D., Pradeep, M.M., Kalpesh, P.O. and Pranavsinh, R.J., (2016). Hyaline fibromatosis syndrome: A rare inherited disorder. Indian Journal of Dermatology, 61(5), . 580.

22. Yap YS, McPherson JR, Ong CK, Rozen SG, Teh BT, Lee AS, Callen DF (2014). The NF1 gene revisited-from bench to bedside. Oncotarget. ;5(15):5873.

23. Filocamo, M. and Morrone, A., (2011). Lysosomal storage disorders: molecular basis and laboratory testing. Human genomics, 5(3),.156.

24. Marques AR, Saftig P.(2019). Lysosomal storage disorders-challenges, concepts and avenues for therapy: beyond rare diseases. Journal of Cell Science Jan 15;132(2).

25. Nampoothiri, S., Yesodharan, D., Sainulabdin, G., Narayanan, D., Padmanabhan, L., Girisha, K.M., Cathey, S.S., De Paepe, A., Malfait, F., Syx, D. and Hennekam, R.C., (2014). Eight years experience from a skeletal dysplasia referral center in a tertiary hospital in Southern India: a model for the diagnosis and treatment of rare diseases in a developing country. American Journal of Medical Genetics Part A, 164(9), 2317-2323.

26. Uttarilli, A., Shah, H., Bhavani, G.S., Upadhyai, P., Shukla, A. and Girisha, K.M., (2019). Phenotyping and genotyping of skeletal dysplasias: Evolution of a center and a decade of experience in India. Bone, 120, 204-211.

27. Rehm, H.L., (2013). Disease-targeted sequencing: a cornerstone in the clinic. Nature Reviews Genetics, 14(4), 295-300.

28. Lelieveld, S.H., Veltman, J.A. and Gilissen, C., (2016). Novel bioinformatic developments for exome sequencing. Human genetics, 135(6),603-614.

29. McKenna, A., Hanna, M., Banks, E., Sivachenko, A., Cibulskis, K., Kernytsky, A., Garimella, K., Altshuler, D., Gabriel, S., Daly, M. and DePristo, M.A., (2010). The Genome Analysis Toolkit: a MapReduce framework for analyzing next-generation DNA sequencing data. Genome research, 20(9),1297-1303.

30. Richards, S., Aziz, N., Bale, S., Bick, D., Das, S., Gastier-Foster, J., Grody, W.W., Hegde, M., Lyon, E., Spector, E. and Voelkerding, K., (2015). Standards and guidelines for the interpretation of sequence variants: a joint consensus recommendation of the American College of Medical Genetics and Genomics and the Association for Molecular Pathology. Genetics in medicine, 17(5),405-423.

31. https://www.omim.org

\section{Tables}

Tables are not available with this version 